\title{
Factores motivacionales y rendimiento académico en estudiantes de tercer año de la carrera de Psicología de la UNAN-Managua, (segundo semestre 2013). Estudio de casos
}

Dalila Ruíz Montiel

Recepción: 25 - 08 - 2015 / Aceptación: 17 - 11 - 2015

\section{Resumen}

El presente trabajo analiza la motivación que en el ámbito de la enseñanza-aprendizaje tuvieron los estudiantes del tercer año de Psicología de la UNAN-Managua, durante el segundo semestre 2013. Se hace referencia a factores que incitan al estudiantado por interesarse en hacer preguntas, participar de forma activa en clase; realizar las actividades propuestas, ejercitarse con las técnicas adecuadas de estudio, la investigación y realización de campo o experimentales.

El objetivo propuesto consistió en el análisis de los factores motivacionales y rendimiento académico obtenido por los estudiantes objeto de estudio, durante el período seleccionado. Se utilizó el enfoque cualitativo para el estudio de casos múltiples. De igual manera, es una investigación descriptiva y de corte transversal.

Para la recolección de la información, se utilizaron instrumentos tales como, grupos focales (se realizaron dos). De igual manera, se realizaron entrevistas focalizadas a cinco estudiantes, con el fin de conformar la muestra. Por último, se consultó el acta de calificaciones para obtener los promedios académicos.

Otras técnicas de recopilación utilizada, fue la utilización de dos informantes claves y tres expertos, a los que se les realizó de forma individual una entrevista focalizada, permitiendo registrar en forma directa sus experiencias y opiniones respecto al tema. Para el procesamiento de la información, se utilizó el análisis de contenido, permitiendo la triangulación de la misma.

Palabras claves: Motivación, Enseñanza-aprendizaje, Rendimiento académico

\begin{abstract}
This paper analyzes the motivation in the field of teaching and learning of students of the third year of Psychology at UNAN - Managua during the second semester 2013. It refers to factors that encourage students to be interested in asking, participate actively in class, complete the proposed activities, study with the adequate techniques and participate in investigate and fieldwork or experimental.
\end{abstract}

The proposed objective was to analyze factors of motivation and academic record during the period, using the qualitative approach during multiple cases approaches, At the same time is an descriptive research of transversal approaches,

Keywoords: Motivation, Teaching and learning, academic performance 


\section{Metodología aplicada}

Los niveles de aprendizaje y rendimiento académico logrados por un educando(a) dependen de múltiples factores, entre ellos la motivación. En el presente estudio se hace énfasis en el análisis de los factores motivacionales y rendimiento académico, en estudiantes de tercer año de la carrera de Psicología de la UNAN-Managua durante el segundo semestre del año 2013.

El enfoque utilizado en la presente investigación es de tipo cualitativo, por ser un proceso que se realiza de manera organizada, sistemática y constituida por etapas o fases. En palabras de Ruíz (2012) los métodos cualitativos son los que permiten conocer la realidad desde una perspectiva de "insider", lo cual consiste en captación del significado particular, que a cada hecho atribuye su propio protagonista y de contemplar estos elementos como piezas de un conjunto sistemático.

Al mismo tiempo, este estudio constituye en una especie de "tesis" socioeducativa cualitativa, ya que en ella se comparten vivencias en el contexto escolar y social donde se desenvuelven las participantes.

El abordaje de la investigación se efectúo por medio del estudio de casos múltiples facilitando la identificación de los factores motivacionales y rendimiento académico de las participantes. Esta dinámica es planteado por Chetty (1996) en Martínez (2006), donde señala que, "con este método los datos pueden ser obtenidos, desde una variedad de fuentes: documentos, registros de archivo, entrevistas directas, observación directa y participante" (p167).

La estrategia de muestreo utilizada en esta investigación, fue de variación máxima, buscando con ello la recolección de toda la variedad decasos posibles. Este tipo de técnica empleada, tenía el propósito de seleccionar una muestra heterogénea representativa del fenómeno estudiado, tomando en cuenta las diferentes características sociodemográficas, personales en los diferentes niveles de rendimiento académico.

Una limitante en este estudio -si se ve en relación al sexo-- tiene que ver con la población universitaria de la carrera de Psicología, la cual está constituida en su mayoría por mujeres, por tanto recoge el sentir de este sector. Los pocos estudiantes varones manifestaron poca disponibilidad a participar en la investigación.

Atendiendo a la explicación anterior y empleando la metodología cualitativa, la muestra se elaboró seleccionando a cinco jóvenes del sexo femenino, incorporando en calidad de colaboradores a dos informantes claves y tres expertos. Para la recolección de información, se empleó las técnicas de grupo focal, entrevista focalizada y el método de revisión documental. Para el procesamiento de la información, se recurrió a la técnica de análisis de contenido, lo que permitió la triangulación de datos a través de matrices categoriales.

\section{Resultados}

Los resultados del estudio sugieren que los factores motivacionales pueden ser internos o externos al estudiantado. Algunos influyeron de manera positiva en el proceso de enseñanza-aprendizaje y rendimiento académico. De manera contraria, se constató que ciertos factores incidieron negativamente. A la vez los expertos conocieron formas significativas de expresión motivacional en los discentes.

\section{Factores motivacionales externos}

Los factores motivacionales externos permiten al educando desarrollar un aprendizaje óptimo o bien pueden ir en detrimento del mismo. Dentro de la 
investigación realizada, se pueden constatar que los factores motivacionales externos no pueden separarse del proceso de enseñanzaaprendizaje. Entre esosfactores, se encuentran los relacionados con los recursos económicos, responsabilidades socio familiares, uso del tiempo, períodos de clases, comportamientos del docente, metodología y estrategias de enseñanza empleadas por los mismos.

En cuanto a los factores motivacionales internos que incidieron en el proceso de enseñanza-aprendizaje se señalan los siguientes: motivación académica e interés por la carrera, deseo de superación, estrategias de aprendizaje y estado de salud.

Al abordarse el factor económico, las y los estudiantes participantes en los grupos focales como los de la muestra, señalan que la disponibilidad de dinero en efectivo, medios tecnológicos, facilidad para su adquisición, entre otros, son recursos indispensables para el desarrollo de la vida estudiantil. En la información obtenida en uno de los grupos focales, se destacó que los recursos económicos juegan un papel importante para el mantenimiento de los estudios. Al respecto una participante sugiere: "Un estudiante puede reprobar cuando le falta el dinero." (Solange, grupo focal, 02 de junio de 2014).

En efecto, los recursos económicos permiten optimizar en alguna medida el aprendizaje, tanto dentro como fuera del salón de clases, dado que si se goza de ciertas condiciones, el alumnado puede tener mayor disposición y preparación para mostrar un mejor desempeño académico. En relación a esta idea, una joven manifestó que, "Para lograr el éxito es indispensable disponer de recursos" (Melody, grupo focal, 02 de junio de 2014).

Como se puede apreciar, la perspectiva planteada por Melody es discutible. Por una parte, se reconoce que tanto la disponibilidad como las limitaciones de recursos económicos determinan ciertas situaciones en la vida de las y los universitarios. De igual manera, es cierto que el éxito educativo no depende únicamente de los recursos económicos que posea el o la estudiante. Este último criterio es señalado por dos participantes entrevistadas, Yahori dice: "He tenido dificultades económicas, pero de alguna forma busco la manera de estudiar." (Yahori, entrevista focalizada, 16 de julio de 2014). Por su parte Olga dice: "Hay limitaciones o carencias (...) pero he sabido equilibrar esos aspectos." (Olga, entrevista focalizada, 17 de julio de 2014).

Es bueno señalar que las dificultades financieras enfrentadas por los estudiantes, no les impiden estudiar, por el contrario representa un reto y un estímulo a ser mejor para que al formarse pueda lograr mejoras económicas para continuar. La universidad a la que asisten las participantes que componen la muestra de la investigación, es de carácter público; se requieren algunos gastos adicionales que los estudiantes deben cubrir. En este sentido, una estudiante expresó: "No tengo computadora, entonces voy a la biblioteca, pero el tiempo que me facilitan es insuficiente." (Solange, grupo focal 02 de junio de 2014).

En relación a los materiales didácticos, de reproducción, útiles escolares, transporte, alimentación y otros gastos, son asumidos por ellas/ ellos, o por sus familias. Pese a estas dificultades los estudiantes de la carrera de psicología no desean abandonar sus estudios ya que consideran que la oportunidad de haber ingresado a la carrera que deseaban para formarse profesionalmente, es un aliciente para continuar.

Encuanto al factor relacionadoconla situación familiar y su entorno, es seguro que la actitud y/o visión que los padres o familiares cercanos tengan o transmitan a sus hijos, respecto a la educación, cultura, profesores y centros educativos influye en el proceso de aprendizaje. Un pensamiento recurrente entre las estudiantes de la muestra, es lograr 
ser el orgullo de sus padres, estableciendo una relación de proyección que vincula los resultados alcanzados por ellos en el campo educativo, como parte de los esfuerzos realizados por sus progenitores. De igual manera, ese sentimiento de gratitud hacia la familia, se convierte en un motivo para mostrarse más interesado (a) en los estudios. Sobre este aspecto Akira señala: "Me motiva que mi mamá, papá vean los frutos y me apoyen y ser el orgullo de ellos ya que se expresan bien delante de sus compañeros de trabajo."(Akira, entrevista focalizada, 17 de julio 2014).

En algunos casos, el ingreso de estas jóvenes a la universidad obedece a un plan familiar y a esfuerzos conjuntos, por tanto el agradecimiento se asocia con sentimiento de orgullo y deseo de honrar las demandas de sus seres queridos. El hecho de ser las únicas personas de la familia que alcanza logros académicos, aumentan los niveles de compromiso entre las estudiantes.

De acuerdo a lo señalado, es importante destacar que el apoyo, la confianza y el esfuerzo de los padres, en relación a la educación de sus hijas, resulta esencial en la motivación de las mismas hacia el proceso de enseñanza aprendizaje. Además ser la primera universitaria de la familia genera sentimiento de orgullo. Por el contrario, cuando la familia desestima la educación, ésta se convierte en un factor de desmotivación.

Como se ha explicado en líneas anteriores, el apoyo familiar contribuye de forma favorable a la motivación, no obstante existen otras situaciones relacionadas con la familia, que también ejercen influencia positiva o negativa en el alumnado. Los factores familiares, también pueden incidir de forma positiva o negativa en el proceso de enseñanza aprendizaje.

Bennett (2014), señala la existencia de factores familiares, que pueden interferir el desarrollo académico y la conducta de los y las estudiantes. Entre ellos se encuentran la estabilidad económica, los cambios en las relaciones familiares, la actitud de los padres hacia la educación y el abuso o acoso en cualquier tipo de expresión.

Los factores familiares son todas aquellas circunstancias o eventos que suceden al interior de la familia y poseen el poder suficiente para causar efectos positivos o negativos en el comportamiento y actitudes de las y los estudiantes universitarios. En el caso del presente estudio, influyeron en la motivación de las estudiantes aspectos como: el duelo, familias mono parentales, divorcios, dificultad en las relaciones familiares, responsabilidades, así como el cuido de los hermanos menores.

En algunos casos se puede advertir que la familia también es un agente desmotivador, pues en vez de brindar afecto y seguridad al estudiante, le genera preocupación y ansiedad, bien sea porque las relaciones entre sus miembros son friccionadas productos de contradicciones, desavenencias, o debido a roles familiares confusos o no suficientemente delimitados. Sobre este aspecto un estudiante que participó del grupo focal afirmó: "Se necesita un ambiente familiar adecuado y sobrellevar los conflictos en la relación de pareja". (Ángel, grupo focal, 02 de junio de 2014).

De igual manera un experto, reafirma lo expuesto con anterioridad: "Un factor des motivacional para el proceso de enseñanzaaprendizaje es cuando la familia, no bien constituida, es disfuncional." (Informante clave 2, entrevista focalizada, 17 de julio de 2014).

Los eventos traumáticos como la muerte de un familiar, accidentes fortuitos vividos al interior del hogar (incendio, inundación o derrumbe), enfermedad crónica de un pariente cercano, entre otras dificultades, suelen tener un fuerte efecto desmotivante en los discentes debido a la conmoción y 
cambios bruscos experimentados en su esfera emocional. Ellos y ellas consideran que es muy difícil rehacer su vida, sintiéndose al mismo tiempo, incapaces de continuar con sus proyectos personales, tal como se reflejan en los siguientes comentarios de Estrella y Dana: (...) "Además murió mi papá." (Estrella, grupo focal, 02 de junio de 2014); “Mi abuelito falleció (...) fue muy fuerte, yo me sentía devastada (...) y me afectaba en mis clases y emocionalmente." (Dana, grupo focal, 02 de junio, 2014).

Es un hecho que los sucesos acontecidos dentro de la familia, muerte de un ser querido o disfuncionalidad de la familia, influyen de forma significativa en la vida de estas estudiantes, afectando su desempeño en clases y desmotivándolas en el proceso de enseñanza-aprendizaje. Se confirma entonces lo planteado por Bennet (2014), quien sostiene que las relaciones en familia, pueden interferir el desarrollo académico y la conducta de los estudiantes.

Para culminar con el abordaje de los factores extrínsecos (externos) es importante mencionar que en el proceso de enseñanzaaprendizaje el comportamiento suscitado por la o el profesor, dentro del salón de clases, determina aprendizajes positivos o negativos de la materia que se reciba. Tradicionalmente en la universidad, como en otras instituciones educativas, se ha cultivado una vieja idea de que el profesor (a) es dueño del conocimiento y la o el estudiante los receptores $\mathrm{y} / \mathrm{o}$ asimiladores de los contenidos.

La dinámica interpersonal y otros elementos que surjan entre ambas partes dentro del salón de clase y las estrategias metodológicas aplicadas, pueden influir en la capacidad del discente para asimilar y aplicar los conocimientos. Un hallazgo interesante en este estudio, es la influencia del comportamiento del docente en la motivación de las y los educandos. En el análisis de este factor, sobresalieron los siguientes aspectos: vocación, motivación, estado de ánimo y su relación afectiva con el alumnado, así como las actitudes mostradas dentro del salón de clases.

Al respecto, López (2007), señala que el profesorado universitario posee fortalezas y debilidades. En las fortalezas, se abordan una variedad de aspectos y parámetros, en especial, aquellos que pasan por el componente vocacional y de motivación que posee el docente. En relación a este tópico, uno de los informantes claves expresó: “El maestro que tiene vocación a la enseñanza, trasmite esa motivación a los estudiantes." (Informante clave 2, entrevista focalizada 17 de julio, 2014).

Por otra parte, las debilidades están referidas a la dificultad del docente para establecer estrategias de motivación entre el alumnado en relación a la asignatura que imparte, circunstancia que genera desmotivación en éstos (López, 2007). Constatando la teoría de López citaremos a Alicia: "De tantos años de dar clase ya se aburrió [el docente]." (Camila, entrevista focalizada, 17 de julio de 2014). Sobre este mismo aspecto, Alicia señala: "Nunca vi nada innovador [en el docente]..." (Alicia, entrevista focalizada, 17 de julio de 2014).

Es posible que la explicación a esta situación, se oriente a que él o la profesora, tal vez no han logrado fomentar la participación activa en sus estudiantes, o porque no sabe cómo transmitir el conocimiento. Estas reacciones nos llevan a pensar que el estado de ánimo de la o el docente, al momento de impartir clases, incide en la manera de enseñar, así como en la motivación de los educandos hacia la materia.

El o la docente es un ser humano y como tal puede experimentar distintos estados de ánimo, según las circunstancias de vida en que se encuentre. Pese a ello, es preciso dejar claro, que la persistencia en algunos de estos estados puede limitar o afectar el desempeño profesional. 
Ser capaz de elegir el estado de ánimo que más se adecúe a una situación, es una de las habilidades más difíciles de desarrollar en el campo de la inteligencia emocional. Que la o el maestro pueda elegir el estado de ánimo correcto, al momento de desarrollar la asignatura, permite ayudarle a controlar cualquier situación en la que se encuentre dentro del salón de clase o en los diferentes ambientes de clase.

En cuanto al vínculo afectivo que se puede establecer entre estudiantes y profesores, Arríen (2013) destaca que el educador y educando están abiertos a apreciarse mutuamente. Esto significa que el proceso educativo es una práctica específica del ser humano, porque en educación se trabaja con personas no con objetos o cosas.

Aunque el planteamiento arriba expuesto es válido y observable en los otros sub sistemas educativos, como la primaria y la secundaria, en donde la o el docente manifiesta de manera abierta su afecto hacia sus alumnos (as) y viceversa; en el contexto de la educación superior o universitaria, puede percibirse cierto escepticismo en algunos docentes. Sobre este aspecto Alicia señala: "Hay algunos que dicen, soy maestro de ustedes, no su amigo..." (Alicia, entrevista focalizada, 17 de julio de 2014).

Contrario a lo anterior, las estudiantes tienen como expectativas que el maestro sea más afectivo y que actúe como un aliado. Así pues, lo manifiesta una de las participantes: "Lo ideal no es crear amistad con el maestro, sin embargo es válido adquirir esa confianza." (Olga, entrevista focalizada, 17 de julio de 2014).

En relación a las razones por las que algunos docentes deciden establecer distancia afectiva con sus alumnos, debe ser otro tema de estudio por la complejidad de elementos que intervienen en este comportamiento. Quizás el mostrarse cariñosos (as) pudiera hacerles sentir que pierden autoridad o control del salón de clase. De igual manera no debe de descartarse reminiscencias de viejos hábitos de enseñanza, vertical negando contacto alguno con los estudiantes.

Según Arríen (2013), la actividad docente de la que el discente no se separa, es por naturaleza aquella experiencia sensible, alegre, emocionante, de mucha carga afectiva. Es falso concebir que la seriedad del docente incompatible con la alegría y el afecto, como si estas emociones fueran enemigas del rigor y la disciplina científica que implica el ejercicio académico.

Lo cierto es que entre personas que conviven cotidianamente y se reúnen para compartir experiencias, es común que surja el aprecio y la simpatía luego de un tiempo de relación, siendo en sí mismo un evento natural entre estudiantes y maestros. En este sentido, si la o el profesor permite un acercamiento con sus estudiantes, contribuirán en gran medida a disminuir la distancia emocional que la jerarquía (y la tradición) establece en la relación docente-discente.

Conviene subrayar que ciertas opiniones dadas a conocer en el marco de este estudio, sugieren que la relación afectiva entre docente y estudiante debe experimentar ajustes que respondan a las expectativas del alumnado. Es pertinente señalar que de forma sutil los participantes en este estudio, identifican cambios en maestros que hacen esfuerzos por establecer nuevas relaciones con los estudiantes.

\section{Factores internos}

En relación a los factores motivacionales internos, se puede afirmar que provienen del placer que se obtiene de la propia tarea o de la satisfacción de la realización de la misma. En palabras de Carrasco (2004) la motivación intrínseca se experimenta cuando al realizar una acción se obtiene satisfacción, sin recibir estimulo del exterior. 
Cuando un discente está motivado intrínsecamente no se necesitan incentivos o castigos, porque la actividad es gratificante en sí misma. Eso se observa por ejemplo, cuando al discente le gusta estudiar, además se complace en participar, sobresalir y ser el mejor. Esto se evidencia en lo manifestado por Estrella estudiante del tercer año de psicología: (...) "Aquellos factores que nos impulsan a seguir adelante y lograr nuestras metas." (Estrella, grupo focal, 02 de junio de 2014). De igual manera lo reafirma Dolores de ese mismo año quien señala que, "La motivación es lo que nos inspira a realizar algo." (Dolores, grupo focal, 02 de junio de 2014).

Como se puede apreciar en las expresiones anteriores, este tipo de motivación interna facilita el aprendizaje porque permite satisfacer las necesidades intelectuales en límites justos, aumenta el conocimiento, y sobretodo hace que el estudiante disfrute de todo el proceso de aprendizaje logrando sus metas. Desde la perspectiva del enfoque humanista, motivar significa activar los recursos internos de las personas, su sentido de competencia, autoestima, autonomía y autorrealización. Esto le permite a la persona escoger lo que es mejor para sí misma; conocerse y actuar en base a estas premisas forman parte de las expectativas del individuo para alcanzar una meta.

Resumiendo lo anterior, se puede señalar que la motivación interna es un proceso psicológico que impulsa a los y las estudiantes a proceder en función de sus metas y necesidades. Este impuso, tiene la fuerza necesaria para enfrentar los retos en la educación superior, evidenciados en la calidad de las acciones y por ende en su rendimiento académico.

Dentro de los factores motivacionales internos que inciden en el proceso de enseñanza aprendizaje y rendimiento académico, se analizan los siguientes: La motivación académica, la motivación hacia la carrera, deseo de superación y salud.
Ausubel (1983) citado por Núñez (2009) destaca que el aprendizaje toma un nivel de significación en las y los estudiantes, solo cuando él o ella poseen capacidad de decisión sobre qué acciones deben de realizarse para generar su propio aprendizaje, retomando conocimientos previos, nivel de compromiso y disposición. De ahí que, la motivación académica ayuda a explicar gran parte del comportamiento del estudiante en el aula y su trascendencia en el proceso enseñanzaaprendizaje.

Esta motivación académica hace posible que la o el estudiante dirija su conducta de forma consciente, hacia las acciones que contribuyan a su formación. Como muestra de lo señalado en el párrafo anterior, es pertinente citar las palabras de Sofía, estudiante de Psicología: (...) "Si no estás motivado, lógicamente no vas a salir bien en clases." (Sofía, grupo focal, 02 de junio de 2014) (Martínez, Nicaragua y Ruiz, 2014).

Se puede mencionar otro factor, aunque pareciese similar al anterior, no es lo mismo: Motivación hacia la carrera. Cuando la motivación se orienta hacia una carrera, implica mayor compromiso personal, ya que conlleva a tomar una decisión sobre la opción profesional que un estudiante puede debe asumir como parte de su proyecto de vida. En este sentido, Camila participante en este estudio, señala: "Uno de ellos es el trabajar más con niños y ayudarlos de alguna manera, tener un preescolar o trabajar en uno, porque me encantan los niños o si no trabajar en comunidades..." (Camila, entrevista focalizada, 17 de julio de 2014). Por su parte Akira afirma: "Me enamoré de la carrera..." (Akira, entrevista focalizada, 17 de julio de 2014) (Martínez, Nicaragua y Ruiz, 2014).

De las anteriores opiniones de las estudiantes se interpreta un sentimiento de identificación y complacencia con la profesión seleccionada, representando una ventaja para sentirse más motivadas y asumiendo compromiso con el estudio. Por consiguiente, se puede inferir 
que la motivación hacia la carrera puede tener connotaciones positivas en el desempeño del estudiante, cuando estudian la carrera que realmente desean.

Ahora bien, existe la posibilidad de que en algunos casos el éxito educativo de los mismos no se alcance, debido a una elección equivocada de lo que deseaban, desde el puno de vista de su desarrollo profesional. Es oportuno mencionar que la motivación académica ayuda a explicar gran parte del comportamiento del discente en el aula y su trascendencia en el proceso enseñanzaaprendizaje. Sobre este aspecto del factor, el Informante clave 1 (docente) plantea: "Se necesita saber qué le gusta, cuál es el perfil profesional que se está formando." (Informante clave 1, entrevista focalizada, 10 de agosto de 2014). Por su parte el docente, Informante clave 2, opina que, "Hay otros factores que inciden en los muchachos, por ejemplo, si la carrera no era primera opción." (Informante clave 2, entrevista focalizada, 17 de Julio de 2014) (Martínez, Nicaragua y Ruiz, 2014).

En definitiva, la motivación hacia la carrera es una condición significativa para el desarrollo pleno, tanto de capacidades como de habilidades propias de lo que se estudia, disfrutando del aprendizaje. Otro factor motivacional interno que puede incidir en el proceso de enseñanza aprendizaje y rendimiento académico, es el grado de deseo de superación que tenga el estudiantado.

En relación al deseo de superación, permite la visualización del futuro desde múltiples direcciones. Esta condición apunta hacia el bienestar económico generado por actividad laboral en las diversas etapas de su vida, ser el orgullo familiar, tener independencia e inclusive graduarse de la universidad. Sobre este aspecto, la estudiante Estrella expresa: "(..) quiero lograr las metas propuestas." (Estrella, grupo focal 02 de junio 2014). (Martínez, Nicaragua y Ruiz, 2014).
Los estudiantes con alta necesidad de superación, poseen fuerzas impulsoras que activan sus deseos para hacer mejor las cosas y funcionar de manera eficaz, demostrando responsabilidad ante el desempeño, lo que es atribuible al éxito y no a la suerte. Esto quiere decir, que los resultados dependen de las decisiones y esfuerzos que toman los estudiantes, en consideración con aquellos que creen en el destino.

La necesidad de buenos resultados en términos académicos, hace que se trabaje más y mejor. A medida que los discentes van alcanzando sus objetivos, se sienten motivados a ser productivos e innovadores, desarrollando afectividad hacia lo que hacen. La estudiante Dana expresa: "Cuando uno está interesado en algo, hace todo lo posible y da todo lo que tenemos (...) es dar la fuerza que tenés." (Dana, grupo focal, 02 de junio de 2014). En ese mismo tono, Camila, expresa: "Quiero ser psicóloga (...) Tengo que ser buena alumna." (Camila, grupo focal, 02 de junio de 2014). Por su parte Yahori refuerza el criterio del éxito con esfuerzo y disciplina: "Creo que me esforcé muchísimo, le he dedicado mi tiempo pero siento que puedo dar más para alcanzar el éxito, ser autodidacta." (Yahori, grupo focal 02 de junio de 2014) (Martínez, Nicaragua y Ruiz, 2014).

De lo anterior descrito, se evidencia que las y los estudiantes que poseen fuertes aspiraciones, se sienten obligados a lograrlas. Están convencidas que el esforzarse con sus estudios y tomando decisiones efectivas pueden concretar sus deseos de superación.

Por último y no menos importante está la salud, factor que incide también en la motivación de los discentes. El diccionario de la Real Academia Española define la salud como la condición física y mental en que se encuentra un organismo en determinado lapso de tiempo. También es entendida como el estado en que un ser orgánico ejerce de manera normal todas sus funciones. 
De lo anterior se puede decir que es lo concerniente a las tareas que normalmente desarrollan las personas en los ámbitos laborales, familiares, de pareja, recreativos, académico, etc. Asimismo, cuando un organismo se encuentra enfermo, la capacidad merma, razón por las cuales las y los discentes que experimentan problemas de salud, bien sea de carácter físico o mental, no presentan las condiciones adecuadas para interesarse en el proceso de enseñanzaaprendizaje.

Desde un malestar común como el dolor de cabeza o menstrual, hasta el padecimiento de una enfermedad crónica, tienen la potencialidad de interrumpir o afectar los procesos mentales y psíquicos, considerados indispensables para el aprendizaje y sus resultados reflejados en su rendimiento académico. Sobre esto, Perla (2008), manifiesta que los estudiantes universitarios se ven sometidos a muchas presiones a lo largo de su carrera, por tanto algunas enfermedades o dolencias comienzan a aparecer.

Afecciones como debilidad, dolor, fatiga, se relacionan con la falta de concentración, lenguaje pausado o poco fluido; poca capacidad para la retención de información, indisposición y desánimo en el salón de clases. Alicia así lo confirma: "Padezco de migraña, los dolores de cabeza de manera periódica, fuertes. A la hora de estudiar me duele." (Alicia, entrevista focalizada, 17 de julio de 2014).

Según Perla (2008) los estudiantes se estresan por la saturación de trabajos y otras preocupaciones relacionadas con su carrera, de ahí que están expuestos a sufrir de estrés y otras enfermedades como gastritis nerviosa o migraña. En este sentido, Akira confirma esta condición y la afectación que le produce en la dinámica de estudio: "Anduve con dolor de cabeza no voy a prestarle atención al profesor." (Akira, entrevista focalizada, 17 de julio de 2014) (Martínez, Nicaragua y Ruiz, 2014).
También se debe considerar el caso de estudiante con enfermedades graves que les impide asistir a clase. Esta situación incide en sus estudios. Mientras el resto de sus compañeros (as) siguen adelante con el proceso de enseñanza-aprendizaje, éste los interrumpe, en contra de su voluntad, de manera que si éste logra recuperarse y decide reintegrarse de nuevo en el estudio, encontrará una serie de dificultades para recuperar el tiempo y actualizarse en cada materia. La colaboradora Yahori, se refiere a este aspecto: "Tuve una infección en los riñones y a partir de eso tuve que faltar a clases, perdí algunas pruebas." (Yahori, entrevista focalizada, 16 de julio de 2014).

A manera de síntesis, la condición de salud es importante en el proceso de aprendizaje y para el rendimiento académico. Cuando las y los educandos pierden la oportunidad de realizar actividades evaluativas o no logran el rendimiento requerido por las exigencias planteadas, debido a una enfermedad, es probable que su rendimiento se vea afectado y se produzca un estado de desmotivación.

\section{Conclusiones}

Es un hecho que la motivación interna permite la consecución de las metas académicas y el aprendizaje de calidad, actuando en los resultados que esperan los discentes y docentes. Por tanto, estos factores motivacionales internos pueden ser determinantes en el proceso educativo, facilitando el desarrollo académico de las y los estudiantes o generando dificultades en el mismo.

Como docentes constructivistas debemos tomar en cuenta las motivaciones internas que mueven o paralizan a nuestros estudiantes en el aula de clase. No es adecuado verle de manera aislada de todo lo que le sucede en su entorno, mucho menos de su mundo interno, si se quiere que los estudiantes egresen con calidad profesional de nuestra Alma Mater. 
La transformación de la educación superior es un proceso arduo sometido a múltiples vicisitudes, donde no debe de descuidarse el factor humano. El comportamiento del o la docente, es un elemento muy importante para el desarrollo de la calidad educativa, ya que de alguna manera facilita los procesos de enseñanza y la concretización de las potencialidades de los y las estudiantes.

En resumen, todos estos factores deben estar orientados a que como docentes comprendamos las necesidades de los estudiantes, mostrando disposición y voluntad hacia la enseñanza. Eso significa "ir más allá de la facilitación de los contenidos", viendo a la persona de forma integral, más que un aprendiz o receptor de conocimientos.

\section{Referencias bibliográficas}

\section{Libros}

- Aebli, Hans (2001). Factores de la enseñanza que promueven el aprendizaje autónomo. Editorial Narcea.

- $\quad$ Alken, Lewis (1996). Test psicológico y evaluación. México: Editorial PrenticeHall Hispanoamericana.

- Bandura, A. (1997).Self-Efficacy: The Exercise of Control. USA: Editorial: Worth Publishers.

- Beltrán, Llera J. (1995). Psicología de la Educación. Barcelona: Editorial Boixareu universitaria.

- Brown, F. (1980).Principios de la Medición en Psicología y Educación. México: Editorial el Manual Moderno.

- Carderera, M. (1965). Principios de Educación y Métodos de Enseñanza. Libro de texto para las escuelas normales. Madrid: Segunda Edición.

- Carrasco, José. (2004).Técnicas y Recursos para Motivar a los alumnos. España: Ediciones Rialp. S.A.

- Cervantes Sanabria, S. (2010) Desarrollo del proceso enseñanza- aprendizaje y su relación con el rendimiento académico en la asignatura de contabilidad de costos específicos en la carrera de contaduría pública y finanzas en el segundo semestre. (Tesis inédita de maestría). Universidad Nacional Autónoma de Nicaragua, (UNAN-Managua).

- Núñez Pérez, José (2009). Motivación, Aprendizaje y Rendimiento Académico. Acta 10 del Congreso internacional gallego- portugués de Psicopedagogía. Universidad de Oviedo.

- Ruiz Olabuénaga, José Ignacio. (2012). Metodología de la investigación cualitativa. Bilbao: Editorial Universidad del Deusto.

\section{Hemerografía}

- $\quad$ Arríen, Juan Bautista. “Afecto y Rigor mueven la relación profesor-discente". El Nuevo Diario. Nicaragua, 05 de julio, 2013. Segmento Opinión. Página 2 A.

\section{Webgrafía}

- Bennett, L.(2014). Los factores familiares que influyen en el comportamiento de los estudiantes en la escuela. Sitio oficial de la Fundación Livestrong.

Recuperado de: http://www. livestrong.com / es / factores familiares-influyen-info_4370/

- Blog spot monyterapias. Definición de autoestima según Virginia Satir.

Recuperado de: http:// mony73comunicacionpareja.blogspot. com/2009/08/la- autoestima-virginiasatir.html.

- Campos, Y. (2003). Estrategia de enseñanza-aprendizaje. Portal de Unidades Temáticas de inglés. English Media.

Recuperado de http://www.cuaed. unam.mx/english_media/descargas/ estrategias_english media.pdf

- Cruz Ramírez, J. (2011). Motivación 
intrínseca e extrínseca. Blog spot Psicólogos en línea Net.

Recuperado de: http://

psicologosenlinea.net/11-motivacionintrinseca- definicion-completa-clases$\mathrm{y}$-factores-de-la-motivacion-intrinseca. $\underline{\mathrm{html}}$

- Diccionario de la Real Academia Española (2015).

Recuperado de: http://dle.rae. es $/$ ?id=X7MRZku.

- López, Walevska (2007). Rasgos determinantes de las aptitudes emprendedoras que forman el perfil de los estudiantes de contaduría pública. Red de Revistas científicas de América
Latina, el Caribe, España y Portugal. Revista de estudios sociológicos. Vol. XXVII, núm. 81, SeptiembreDiciembre, México.

Recuperado de: http://www.redalyc. org/pdf/598/59820678002.pdf

- Martínez, Piedad Cristina (2006). El método de estudio de caso. Estrategia metodológica de la investigación científica. Pensamiento y gestión, 20. Universidad del Norte, 165-193, 2006. Recuperado de: http://ciruelo. uninorte.edu.co/pdf/pensamiento gestion/20/5_El_metodo_de_estudio_ de_caso.pdf 